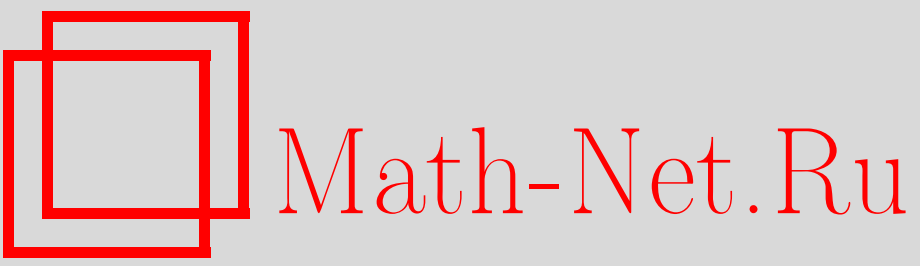

Г. А. Айгунов, Об ограниченности ортонормированных собственных функций нелинейной краевой задачи типа Штурма-Лиувилля с неограниченной сверху весовой функцией на конечном отрезке, УМH, 2002, том 57, выпуск 1, 145-146

DOI: https://doi.org/10.4213/rm477

Использование Общероссийского математического портала Math-Net.Ru подразумевает, что вы прочитали и согласны с пользовательским соглашением

http: //www.mathnet.ru/rus/agreement

Параметры загрузки:

IP : 54.198 .187 .58

26 апреля 2023 г., $11: 22: 08$ 


\title{
ОБ ОГРАНИЧЕННОСТИ ОРТОНОРМИРОВАННЫХ СОБСТВЕННЫХ ФУНКЦИЙ НЕЛИНЕЙНОЙ КРАЕВОЙ ЗАДАЧИ ТИПА ШТУРМА-ЛИУВИЛЛЯ С НЕОГРАНИЧЕННОЙ СВЕРХУ ВЕСОВОЙ ФУНКЦИЕЙ НА КОНЕЧНОМ ОТРЕЗКЕ
}

\author{
Г.А. Айгунов
}

Рассмотрим нелинейную краевую задачу:

$$
\begin{aligned}
& -\left(y^{\prime}(x)\right)_{p}^{\prime}=\lambda x^{-\alpha}(y(x))_{p}, \quad 0<x<1, \\
& y(0)=y(1)=0, \quad \int_{0}^{1} x^{-\alpha} y^{2}(x) d x=1,
\end{aligned}
$$

где $0<\alpha<1, p>1$ и $(z)_{p} \equiv|z|^{p-1} \operatorname{sign} z=|z|^{p-2} z$. В работе доказьвается следующая

Теорема 1. Краевая задача (1)-(2) имеет непрерьвные (вместе с первой производной) на $[0,1]$ собственные функции $y_{k}(x), k \in \mathbb{N}$. Совокупность собственных функций $\left\{y_{k}(x)\right\}$ равномерно по $k$ и $x$ ограничена.

Для доказательства существования совокупности $\left\{y_{k}(x)\right\}$ достаточно показать, что решение задачи Коши с условием $y(0)=0, y^{\prime}(0)=\alpha$ существует и единственно.

Действительно, так как при $x>0 x^{-\alpha}$ - непрерьвная функция, то можно воспользоваться свойствами монотонности нулей [1] и точно таким же образом, как и в указанной работе, показать существование $\left\{y_{k}(x)\right\}$. Справедлива следующая

Лемма. Пусть $\rho(x)>m$ непрерывна на $(0,1]$ и суммируема на $[0,1]$. Тогда существует единственное решение задачи Коши

$$
-\left(y^{\prime}(x)\right)_{p}^{\prime}=\lambda \rho(x)(y(x))_{p}, \quad y(0)=a, \quad y^{\prime}(0)=b \quad \text { при любых вещественных а и } b .
$$

ДокАЗАТЕЛЬСтво. Как показано в [1], при преобразовании $y^{\prime}(x)=r(x) s^{\prime}(\varphi(x)), y(x)=$ $r(x) s(\varphi(x))$ наше уравнение перейдет в систему

$$
\left\{\begin{array}{l}
\varphi^{\prime}(x)=\left|s^{\prime}(\varphi)\right|^{p}+\lambda \rho(x)|s(\varphi)|^{p} \\
r^{\prime}(x)=r(x) s^{\prime}(\varphi)(s(\varphi))_{p}[1-\lambda \rho(x)] .
\end{array}\right.
$$

Так как $\left|s^{\prime}(\varphi)\right|^{p}+|s(\varphi)|^{p}=1$, то $r^{p}(x)=\left|y^{\prime}(x)\right|^{p}+|y(x)|^{p}$.

Поэтому $r(0)=\left(|a|^{p}+|b|^{p}\right)^{1 / p}$. Если $r(0) \neq 0$, то из соотношения $y(0)=r(0) s(\varphi(0))$ определим $\varphi(0)$. Если $r(0)=0$, то $\varphi(0)$ зададим произвольно.

Из второго уравнения системы следует, что

$$
\ln r(x)=\ln c+\int_{x_{0}}^{x} s^{\prime}(\varphi(t))(s(\varphi(t)))_{p}[1-\lambda \rho(t)] d t, \quad \text { где } x_{0} \in(0,1] .
$$

Отсюда $r(x)=c e^{\int_{x_{0}}^{x} s^{\prime}(\varphi(t))(s(\varphi(t)))_{p}[1-\lambda \rho(t)] d t}$; так как $\rho(t)$ суммируема, то интеграл конечен.

Следовательно, либо $r(x)$ нигде не обращается в нуль, либо $r(x) \equiv 0(c=0)$, поэтому решение $r(x)=0$ - единственное в случае $r(0)=0$. Значит, исходная задача Коши имеет единственное решение $y(x) \equiv 0$ в случае $a=b=0$.

Пусть $r(0) \neq 0$. Тогда функция

$$
r(x)=\frac{r(0)}{e^{\int_{x_{0}}^{x} s^{\prime}(\varphi(t))(s(\varphi(t)))_{p}[1-\lambda \rho(t)] d t}} e^{\int_{x_{0}}^{x} s^{\prime}(\varphi(t))(s(\varphi(t)))_{p}[1-\lambda \rho(t)] d t}
$$

будет решением (однозначно по $r(0)$ определенным), если $\varphi(t)$ - решение первого уравнения уже найдено. Таким образом, достаточно показать, что первое уравнение с начальньп условием $\varphi(0) \in\left[-\pi_{p} / 2, \pi_{p} / 2\right]$ имеет единственное решение, $\pi_{p}=2 \pi /(p \sin (\pi / p))$. 


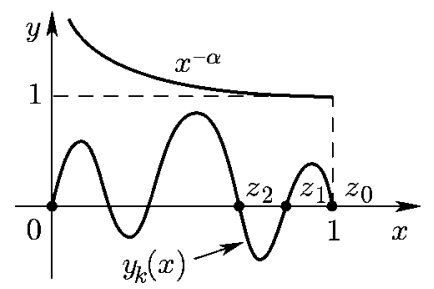

Рис. 1

Переписав первое уравнение в виде $\varphi(x)=\varphi\left(x_{0}\right)+\int_{x_{0}}^{x}\left[\left|s^{\prime}(\varphi(t))\right|^{p}+\lambda \rho(t)|s(\varphi(t))|^{p}\right] d t$, мы видим, что из интегрируемости $\rho(x)$, гладкости и ограниченности $s(\varphi)$ следует существование и единственность необходимого решения.

Лемма доказана и доказано существование $\left\{y_{k}(x)\right\}$, так как $x^{-\alpha}$ - суммируемая функция на $[0,1]$. Покажем теперь ограниченность совокупности функций $\left\{y_{k}(x)\right\}$.

Обозначим нули $y_{k}(x)$ в порядке убьвания через $z_{0}, z_{1}, z_{2}, \ldots\left(z_{0}=1, z_{k}=0\right)$.

Введем также обозначения $\alpha_{i}=\left|y_{k}^{\prime}\left(z_{i}\right)\right|, h_{i}=\max _{x \in\left[z_{i}, z_{i+1}\right]}\left|y_{k}(x)\right|$.

По лемме 1 из [2], учитывая, что $\rho(x)=x^{-\alpha}$, имеем оценки

$$
h_{i} \leqslant \frac{c \alpha_{i}}{\left(z_{i}^{-\alpha} \lambda_{k}\right)^{1 / p}}, \quad 1 \leqslant \frac{\alpha_{i+1}}{\alpha_{i}} \leqslant\left(\frac{z_{i+1}^{-\alpha}}{z_{i}^{-\alpha}}\right) \text { и } h_{0} \geqslant \frac{\bar{c} \alpha_{0}}{\left(z_{1}^{-\alpha} \lambda_{k}\right)^{1 / p}} .
$$

Отсюда $\frac{\alpha_{j}}{\alpha_{0}}=\frac{\alpha_{j}}{\alpha_{j-1}} \cdot \frac{\alpha_{j-1}}{\alpha_{j-2}} \ldots \frac{\alpha_{1}}{\alpha_{0}} \leqslant\left(\frac{z_{j}^{-\alpha}}{z_{j-1}^{-\alpha}}\right)^{\frac{1}{p}}\left(\frac{z_{j-1}^{-\alpha}}{z_{j-2}^{-\alpha}}\right)^{\frac{1}{p}} \ldots\left(\frac{z_{1}^{-\alpha}}{z_{0}^{-\alpha}}\right)^{\frac{1}{p}}=\left(\frac{z_{j}^{-\alpha}}{z_{0}^{-\alpha}}\right)^{\frac{1}{p}}$, или $\alpha_{j} \leqslant z_{j}^{-\frac{\alpha}{p}} \alpha_{0}$, следовательно, $h_{j} \leqslant \frac{c z_{j}^{-\frac{\alpha}{p}} \alpha_{0}}{\left(z_{j}^{-\alpha} \lambda_{k}\right)^{1 / p}}=\frac{c z_{1}^{-\frac{\alpha}{p}}}{\bar{c}} \frac{\bar{c} \alpha_{0}}{\left(z_{1}^{-\alpha} \lambda_{k}\right)^{1 / p}}$ и $h_{j} \leqslant \frac{c z_{1}^{-\frac{\alpha}{p}} \alpha_{0}}{\bar{c}} h_{0}$.

Очевидно, что $z_{1}(k)>z_{1}(2)$ для любого $k>2$, поэтому имеем $h_{j} \leqslant \frac{c z_{1}^{-\alpha / p}(2)}{\bar{c}} h_{0}=c_{0} h_{0}$. Таким образом, если показать, что $h_{0}(k)$ равномерно по $k$ ограничена, то теорема будет доказана. В силу того, что $y_{k}(x)$ нормирована, имеем:

$$
\left\|y_{k}(x)\right\|_{c}=\frac{\left\|y_{k}(x)\right\|_{c}}{\left(\int_{0}^{1} x^{-\alpha} y_{k}^{2}(x) d x\right)^{1 / 2}} \leqslant \frac{c_{0} h_{0}(k)}{\left(\int_{0}^{1} x^{-\alpha} y_{k}^{2}(x) d x\right)^{1 / 2}}<\frac{c_{0} h_{0}(k)}{\left(\int_{1 / 2}^{1} x^{-\alpha} y_{k}^{2}(x) d x\right)^{1 / 2}} .
$$

Последнее выражение равномерно по $k$ ограничено, так как на сегменте $\left[\frac{1}{2}, 1\right]$ функция $x^{-\alpha}$ непрерывна и имеет ограниченную вариацию, а $y_{k}(x)$ можно рассматривать как решение некоторой задачи Коши [3]. Таким образом, мы доказали, что краевая задача (1)-(2) имеет непрерывные на $[0,1]$ собственные функции $y_{k}(x)$, равномерно по всем $k$ и $x$ ограниченные, если $\alpha$ достаточно мало. Справедлива следующая

Teорема 2. Cуществует $\rho(x) \in C_{(0,1]}^{\infty}$ такая, что $0<m \leqslant \rho(x) \leqslant M(0 \leqslant x \leqslant 1) u$ $C_{0}>\varlimsup_{n \rightarrow \infty} \frac{\left\|y_{n}(x, \rho)\right\|_{C_{[0,1]}}}{\lambda_{n}^{\frac{1}{2 p}}}>0$, əде $C_{0}=$ const.

\section{СПИСОК ЛИТЕРАТУРЫ}

[1] A. Elbert // Colloq. Math. Soc. János Bolyai. 1981. V. 30. P. 153-180. [2] Г. А. Айгунов // Изв. вузов. Сев.-Кав. регион. Естеств. науки. 1999. № 1. С. 3-11. [3] Г. А. Айгунов // УМН. 2000. T. 55. № 2. C. 129-130. 